need for dyadic focus on how AA dementia dyads function together.

\section{BURDEN AND FINDING MEANING IN AFRICAN AMERICAN CAREGIVERS FOR INDIVIDUALS WITH DEMENTIA}

S. McLennon, University of Tennessee-Knoxville, Knoxville, Tennessee, United States

Previous research suggests cultural variations in caregivers' perceptions of caring for people with dementia. The purpose of this study was to describe the caregiving experience in a subset of African Americans from a previous study $(\mathrm{N}=14)$. Measures included the Zarit Burden Interview and Finding Meaning through Caregiving (FMCG). Content analysis was performed on narrative comments. Caregivers were primarily female $(64 \%)$, provided care for people with dementia for $>60$ months $(64 \%)$ and had a mean age of 74.6 years. Caregivers reported low burden $(M=31.6)$ and scored high on the FMCG scale (Mean=177.4). Findings from FMCG subscales of Provisional Meaning, Ultimate Meaning, and Loss/Powerlessness have potential to elucidate cultural variations in African American's response to caregiving. Narrative comments were categorized into unmet needs, benefits, and challenges.

\section{DOES A PSYCHOEDUCATIONAL INTERVENTION WITH \& WITHOUT EXERCISE AFFECT SLEEP QUALITY IN AFRICAN AMERICAN CAREGIVERS?}

G. Brewster ${ }^{1}$, M.W. Parker, M.D², F. Epps, PhD $^{3}$, C. Dye, $\mathrm{PhD}^{4}, \mathrm{M}$. Higgins, $\mathrm{PhD}^{5}$,

D.L. Bliwise, $\mathrm{PhD}^{6}$, K. Hepburn, $\mathrm{PhD}^{7}, 1$. Nell Hodgson Woodruff School of Nursing, Emory University, Atlanta, Georgia, United States, 2. Emory Alzheimer's Disease Research Center, Atlanta, GA, 3. Byrdine F. Lewis College of Nursing and Health Professions, Atlanta, GA, 4. Atlanta, GA, 5. Emory University Nell Hodgson Woodruff School of Nursing, Atlanta, GA, 6. Emory University School of Medicine, Atlanta, GA; Emory University Nell Hodgson Woodruff School of Nursing, Atlanta, GA, 7. Nell Hodgson Woodruff School of Nursing, Atlanta, GA; Alzheimer's

Disease Research Center, Atlanta, GA

Caregivers of persons living with dementia report poorer sleep quality compared to non-caregivers. This secondary data analysis examined whether a psychoeducational intervention with and without exercise versus usual care improved caregiver sleep quality. Sleep quality was measured using the Pittsburgh Sleep Quality Index. 142 African American caregivers were randomized: 53 (usual care, UC), 41 (psycho-education, PE), and 48 (psycho-education plus exercise, $\mathrm{PD}+\mathrm{EX}$ ). All caregivers had poor sleep at baseline [mean PSQI: 8.2(UC), 8.8(PE), $8.5(\mathrm{PE}+\mathrm{EX})]$ with no significant difference among the three groups. After six months, caregivers in the UC and $\mathrm{PE}+\mathrm{EX}$ groups experienced a non-significant decline of 0.5 and 1, respectively, in mean PSQI score. However, all the caregivers still had poor sleep [mean PSQI: 7.7(UC), 8.5( $\mathrm{PE}), 7.6(\mathrm{PE}+\mathrm{EX})]$. These results support the need for healthcare providers to assess caregivers for sleep disturbances. It is recommended that caregiver interventions to be inclusive of instructions about and techniques on reducing sleep disturbances.

\section{SESSION 1500 (SYMPOSIUM)}

CULTURE CHANGE PRACTICES IN U.S. NURSING HOME: PREDICTORS OF PRACTICE, AND OUTCOMES ASSOCIATED WITH PRACTICE CHANGE Chair: S.C. Miller, Brown University, Warwick, Rhode Island

Discussant: C.E. Bishop, Heller School for Social Policy \& Management, Brandeis University, Weston, Massachusetts

Given the new U.S. nursing home $(\mathrm{NH})$ regulatory requirements emphasizing person-centered care, depiction of contemporary NH practice is of interest. During this symposium we detail the 2016/17 national prevalence of the processes and structures promoted by the $\mathrm{NH}$ culture change movement and how NH characteristics and Medicaid payment policies are associated with prevalence. Also, we present how resident-centered care, staff empowerment and physical environment practices changed in a nationally representative sample of NHs between the 2009/10 (Time 1) and $2016 / 17$ (Time 2) culture change surveys, factors associated with change, and associations between increased implementation and quality outcomes. The Time-1 survey focused on the critical domains of Environmental Practices (i.e., homelike environment), Resident Care Practices (i.e., resident-centered/-directed care), and Workplace Practices (i.e., staff empowerment) and the Time-2 survey expanded the measures within these domains and also collected data on the additional domains of NH Leadership (i.e., modeling and enabling of culture change) and Family and Community (i.e., inclusion/involvement). In 2016/17, the nationwide survey was administered to $2,142 \mathrm{NH}$ administrators at $\mathrm{NHs}$ previously responding to the Time- 1 survey. The response rate was $74 \%(1,584)$, with no evidence of non-response bias, enabling us to generalize findings to U.S. NHs (using survey weights). This high $\mathrm{NH}$ response rate was critical to the validity of our findings, and factors critical to achieving high $\mathrm{NH}$ response rates will be discussed. Findings show Medicaid pay-for-performance policy is an important predictor of practice prevalence and change, and that increases in staff empowerment scores are associated with improved outcomes.

\section{THE 2016/17 PREVALENCE OF CULTURE CHANGE PRACTICE IN U.S. NURSING HOMES}

S.C. Miller ${ }^{1}$, M.L. Schwartz, $\mathrm{MPH}^{2}$, J.C. Lima, $\mathrm{PhD}^{3}$, R.R. Shield, $\mathrm{PhD}^{4}$, D.A. Tyler, $\mathrm{PhD}^{5}$, C.W. Berridge, $\mathrm{PhD}^{6}$, M.J. Lepore, $\mathrm{PhD}^{7}$, M.A. Clark, $\mathrm{PhD}^{8}, 1$. Brown University, Warwick, Rhode Island, United States, 2. Brown University School of Public Health, Providence, RI, USA, 3. Brown University School of Public Health, Providence, RI, USA, 4. Brown University School of Public Health, Providence, RI, USA, 5. Research Triangle Institute, Boston, MA, USA: , 6. School of Social Work, University of Washington, Seattle, WA, USA: , 7. Research Triangle Institute, Washington, DC, USA; Brown University School of Public Health, Providence, RI, USA, 8. University of Massachusetts Medical School, Worcester, MA, USA

Using 2016/17 survey data, we estimated the national prevalence of the processes and structures promoted by the nursing home $(\mathrm{NH})$ culture change movement. We administered 
the 2016/17 nationwide survey to 2,142 NH administrators at NHs that previously responded to our 2009/10 survey, and the response rate was $74 \%(1,584)$. From responses, we created index scores for the practice domains of physical environment, staff empowerment, resident-centered care, end-of-life care (a subset of resident-centered care), leadership, and family and community engagement. Indices had good internal consistency, and positive associations between index scores and quality indicators supported the criterion validity of the indices. At 82.6 (of 100), the (weighted) standardized index score for resident-centered care practices was the highest, and 76.2 for end-of-life care practices the next highest. Across all domains, there were higher domain index scores in NHs with higher leadership index scores and in states with Medicaid culture change pay-for-performance.

\section{SURVEYING NURSING HOME LEADERSHIP: SUCCESSES AND CHALLENGES}

M.A. Clark ${ }^{1}$, B. Behl-Chadha, $\mathrm{PhD}^{2}$, D. Brown ${ }^{3}$, S. Fish ${ }^{4}$, C. Hillerns ${ }^{5}$, P. Lei ${ }^{6}$, S.C. Miller, $\mathrm{PhD}^{7}, 1$. University of Massachusetts Medical School, Worcester, Massachusetts, United States, 2. University of Massachusetts Medical School, Worcester, MA, USA, 3. University of Massachusetts Medical School, Worcester, MA, USA, 4. University of Massachusetts Medical School, Worcester, MA, USA, 5. University of Massachusetts Medical School, Worcester, MA, USA, 6. University of Massachusetts Medical School, Worcester, MA, USA, 7. Brown University School of Public Health, Providence, RI, USA

We surveyed Nursing Home Administrators (NHA) from 2,142 nationally-representative long-term care facilities about culture change practices with a response rate $(R R)=74 \% \quad(n=1584)$ and no evidence of non-response bias. We first verified contact information for the NHA. Each NHA was then mailed a questionnaire, self-addressed return envelope, cover letter with a user-name and password for web-response if preferred, a letter of support from the two major nursing home associations, and offer of a \$25 gift card for participation. Telephone/e-mail/mail follow-up contacts were initiated with non-respondents two weeks after the initial mailing and tailored thereafter. During follow-up, a telephone option was also offered. Of the completed surveys, $55.9 \%$ were completed by web, $39.6 \%$ by mail, and $4.5 \%$ by telephone. Up to 20 follow-up contacts were made to each facility. Challenges included NHA turnover, time needed for data collection (17 months), and cost ( $\$ 170$ per case; $\$ 240$ per completed interview).

\section{CHANGE IN CULTURE CHANGE PRACTICE IN U.S. NURSING HOMES}

J. Lima ${ }^{1}$, M.L. Schwartz, MPH${ }^{2}$, P.L. Gozalo, PhD. ${ }^{3}$, M.A. Clark, $\mathrm{PhD}^{4}$, S.C. Miller, $\mathrm{PhD}^{5}$, 1. Brown University School of Public Health, Providence, Rhode Island, United States, 2. Brown University School of Public Health, Providence, RI, USA, 3. Brown University School of Public Health, Providence, RI, USA, 4. University of Massachusetts Medical School, Worcester, MA, USA, 5. Brown University School of Public Health, Providence, RI, USA

We collected survey data from 1565 U.S. nursing homes (NHs) in 2009/10 and 2016/17 on three indexes aligned with
$\mathrm{NH}$ culture change. Among NHs without high baseline scores (therefore having potential to improve), $49 \%$ improved over time in their physical score, $40 \%$ in staff empowerment, and $60 \%$ in resident-centered care. NHs in states with payment incentives for performance $(\mathrm{p} 4 \mathrm{p})$ at baseline were significantly more likely to improve in physical and staff empowerment scores compared to NHs in non-p4p states. NHs with higher proportions of black residents and residents with Medicaid were significantly less likely to improve in physical scores. Other NH and market characteristics were not associated with improvement. Among facilities with low index scores at Time 1 , improved staff empowerment scores were associated with lower aide turnover and fewer health and quality of life deficiencies. Improved physical environment and resident scores were not significantly associated with these facility-level outcomes.

\section{USE OF PALLIATIVE CARE CONSULTS AND END-OF- LIFE CULTURE CHANGE PRACTICES IN NURSING HOMES}

M.L. Schwartz 1 , M.A. Clark, $\mathrm{PhD}^{2}$, J.C. Lima, $\mathrm{PhD}^{3}$, S.C. Miller, $\mathrm{PhD}^{4}$, 1. Brown University, Brookline, Massachusetts, United States, 2. University of Massachusetts Medical School, Worcester, MA , 3. Brown Unviersity School of Public Health, Providence RI , 4. Brown Unviersity School of Public Health, Providence RI

Specialty palliative care (PC) provides services similar to hospice without the need for terminal diagnosis. We used 2016/2017 survey data for $1,362 \mathrm{NHs}$ to evaluate whether differences in PC consult use is associated with end-of-life (EOL) specific culture change practices, and assessed the relationship between EOL care and outcomes. EOL care was measured using six questions related to care for dying residents. NHs were categorized as offering: no PC consults, only external consults, or any internal consults. We used logistic regression to evaluate the relationship between consult type and EOL score above the median, controlling for NH structure, staffing, and case-mix. Compared to NHs with no PC consults, the odds of having an EOL score above the median were $1.71(95 \%$ CI: 1.21,2.13) for NHs with only external and 2.19 (95\% CI: 1.55,3.27) for NHs with any internal consults. Better EOL scores were associated with increased hospice use, but not reduced hospitalizations.

\section{SESSION 1505 (SYMPOSIUM)}

\section{SUSTAIN: IMPROVING INTEGRATED CARE DELIVERY FOR OLDER PEOPLE WITH COMPLEX CARE NEEDS ACROSS EUROPE}

Chair: S.R. de Bruin, National Institute for Public Health and the Environment (RIVM), Bilthoven, the Netherlands, de Bilt, Utrecht

Discussant: R. Schreiber, Fallon Health/University of

Massachusetts, Worcester, Massachusetts

Due to population ageing, health and social care systems face the challenge of providing care and support to an increasing number of older adults. Numerous integrated care initiatives have been rolled out in a range of settings and contexts, in order to improve care and support for frail older adults living at home. Despite this rich and varied 